\section{A) Check for updates}

Cite this: Org. Chem. Front., 2022, 9 2084

Received 25th January 2022,

Accepted 27th February 2022

DOI: $10.1039 / d 2 q 000129 b$

rsc.li/frontiers-organic

\title{
Controlling rotary motion of molecular motors based on oxindole $\uparrow$
}

\author{
Daisy R. S. Pooler, (DD) Daniel Doellerer, (D) Stefano Crespi (D) and Ben L. Feringa (D)*
}

Molecular motors are essential components of artificial molecular machines, which can be used to manipulate and amplify mechanical motion at the nanoscale to create machine-like function. Since the discovery of light-driven rotary molecular motors, the field has been widely developed, including the introduction of molecular motors based on oxindole by our group in 2019. The rotational properties of molecular motors, e.g. absorption wavelength, quantum yield and rotation speed, often critically depend on substituent effects. Up to now, the substituent effects of oxindole-based molecular motors have not yet been investigated. Herein, we present a family of oxindole-based molecular motors functionalised at three different positions on the motor core, with either $\mathrm{CN}$ or OMe groups. The motors prepared in this work retain the favourable features of oxindole-based motors, i.e. simple synthesis and visible light addressability. We find that functionalisation has substantial effects on the absorption wavelength of the motors, meanwhile the rotation speed is unaffected. Furthermore, we found that functionalisation of the oxindole molecular motors increases their quantum efficiency considerably in comparison to previous motors of their class.

\section{Introduction}

The development of artificial molecular machines, motors and switches over the past few decades has brought chemists closer to the goal of precisely controlling motion at the molecular level. ${ }^{1-11}$ Light-driven molecular motors are fascinating molecules that can be utilised to impart motion at the nanoscale, and can drive systems out of equilibrium due to their intrinsic ability to undergo unidirectional and repetitive rotation. ${ }^{12}$ Since the first light-driven unidirectional rotary molecular motor presented by our group in $1999,{ }^{13}$ a vast amount of research has been carried out on overcrowded alkene-based molecular motors. ${ }^{14,15}$ They are now highly tuneable actuators which can be tailored to diverse purposes in a multitude of different fields, such as smart materials, ${ }^{16-18}$ catalysis $^{19-21}$ and biomedical applications. ${ }^{22-25}$ Synthetic modification can fine-tune the properties of molecular motors towards these purposes, for example altering their rotational speed or quantum efficiency. ${ }^{15}$ Potentially harmful UV light is commonly used to excite unfunctionalised overcrowded

Stratingh Institute for Chemistry, Zernike Institute for Advanced Materials, University of Groningen, Nijenborgh 4, 9747 AG Groningen, The Netherlands. E-mail: b.l.feringa@rug.nl

$\dagger$ Electronic supplementary information (ESI) available. CCDC 2143239, 2143240 and 2143164. For ESI and crystallographic data in CIF or other electronic format see DOI: $10.1039 / \mathrm{d} 2 q 000129 \mathrm{~b}$ alkenes ${ }^{15,26}$ hence, a substantial research effort currently aims to shift the absorption wavelength of molecular motors towards the visible region of the electromagnetic spectrum. This goal can be achieved through integration of extended $\pi$-systems, ${ }^{27-29}$ push-pull substituents ${ }^{30,31}$ and photosensitisers. ${ }^{32,33}$ Novel molecular motor scaffolds have also been developed towards this end, including imine-based molecular motors by the group of Lehn, ${ }^{34,35}$ and hemithioindigo-based motors introduced by Dube and co-workers. ${ }^{36-39}$ More recently, our group has developed a new class of molecular motors based on oxindole, which are readily synthesised and driven by visible light. ${ }^{40,41}$ The rotation speed of oxindole-based molecular motors can be easily tuned by condensing upper halves with varying steric demand to the oxindole lower half, and due to the heterocyclic oxindole moiety the motors have inherently red-shifted absorption wavelengths. ${ }^{15}$ Further red-shifting of these motors may be achieved through substitution of the motor backbone with various functional groups. Oxindole motors may also suffer from low quantum yields, between $2-3 \%{ }^{40}$ Functionalisation at positions in direct resonance with the central alkene axle has been shown to greatly affect quantum yields of motor rotation..$^{41,42}$

Here, we describe a family of oxindole motors fitted with electron-withdrawing and -donating substituents at three different positions of the motor core (Scheme 1A). The $\mathrm{R}^{1}$ and $\mathrm{R}^{2}$ sites were selected as they are directly conjugated with the central olefinic axle of rotation, and therefore are expected to 
(A)

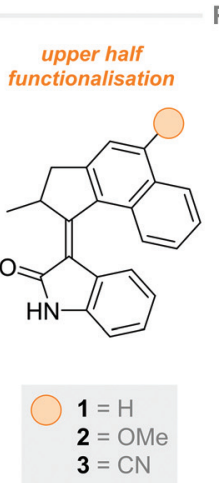

Prepared oxindole motors

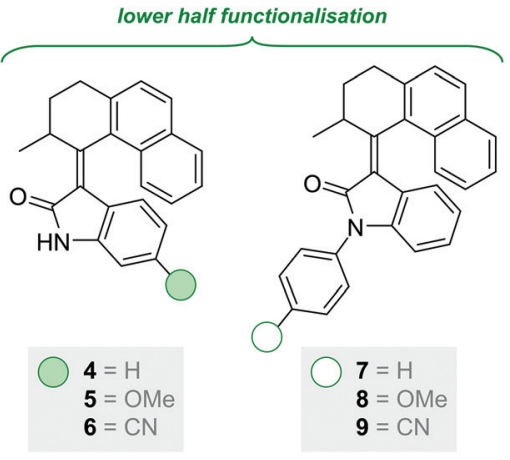

(B)
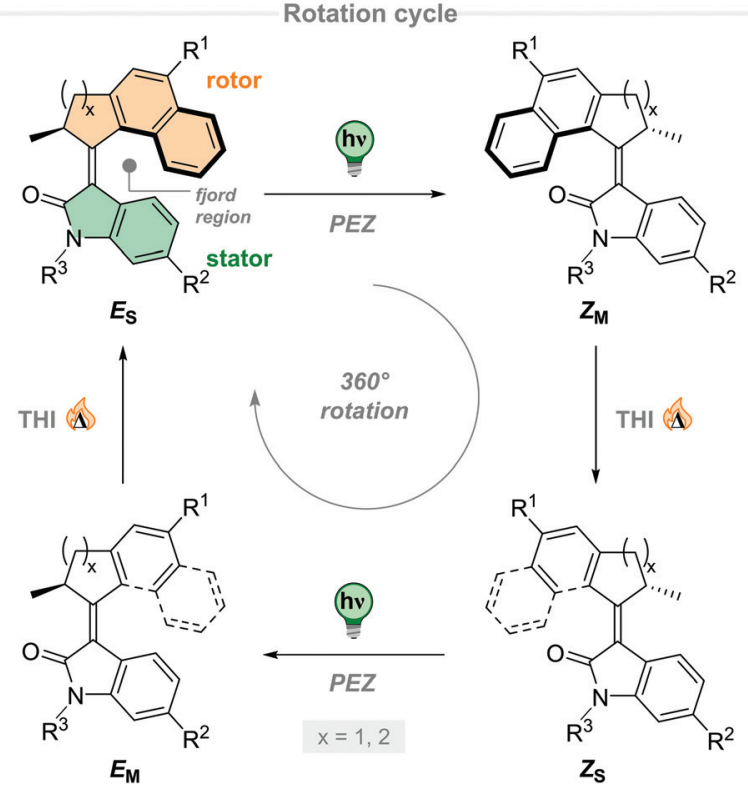

THI (A)

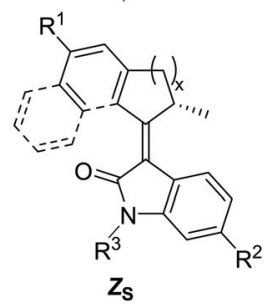

Scheme 1 A) Oxindole-based molecular motors 1-9 investigated in this study; (B) four-step rotation cycle of the motors.

have major influence on the photochemical and thermal properties of the motors. ${ }^{43}$ The $\mathrm{R}^{3}$ site offers conjugation to the oxindolic amide and shows potential as a convenient anchoring point of the motor for integration into larger functional systems. The effect of using different upper halves on the rotational properties of oxindole motors is described in our original work, ${ }^{40}$ however substituent effects have not yet been investigated. We expect electron-withdrawing and -donating groups to influence the absorption wavelength of the oxindole motors, potentially red-shifting them further into the visible region of the electromagnetic spectrum..$^{30,43}$ We chose to investigate $\mathrm{CN}$ and OMe groups as electron-withdrawing and -donating groups, respectively, as they have been shown to be tolerable functional groups in fluorene-based molecular motors. ${ }^{30,42}$

To this end, we developed motors 1-3 based on a 5-membered naphthalene upper half, with the substituents in conjugation with the central olefinic bond. The five-membered ketones with functionalisation in the 6-position were more synthetically viable than the six-membered ketones, making them more readily accessible which will facilitate utilisation of the resulting motors for various applications. Motors 4-9 were fitted with a six-membered ring naphthalene-derived upper half, as it is reported that oxindole motors with this core have thermal half-lives in the region of multiple days. ${ }^{40}$ The novel oxindole lower half provides an attractive handle for further functionalisation; we decided to focus our attention on substitution at the 5-position of the oxindole, due to its interaction with the central double bond through resonance, affording motors 4-6. Unlike our previously reported oxindole motors ${ }^{40,41}$ the current design features a non-methylated amide group on the oxindole lower half, which can be further functionalised to generate $N$-arylated oxindole motors 7-9.

All of the new motors 1-9 follow a four-step rotation cycle typical of oxindole-based molecular motors, consisting of two endergonic photochemical $E-Z$ isomerisation (PEZ) steps and two exergonic thermal helix inversion (THI) steps (Scheme 1B, vide infra). ${ }^{40,41}$

\section{Results and discussion}

\section{DFT calculations}

DFT calculations were performed to predict the structural parameters, relative energies of the THI steps, and absorption wavelengths of oxindole motors 1-9. All structures were optimised using the $\omega$ B97X-D functional with the def2-SVP basis set. The SMD solvation mode ${ }^{44}$ with DMSO as the solvent was chosen, as previous calculations and studies on oxindole motors were carried out in DMSO. ${ }^{40}$ Optimisations were also carried out in the gas phase, and in $\mathrm{CH}_{2} \mathrm{Cl}_{2}$ and $\mathrm{MeOH}$ (see ESI†).

The calculated relative energy barriers for both of the THI steps in DMSO are presented in Table 1. Similarly to previously reported oxindole motors, ${ }^{40}$ motors 1-3 with a five-membered ring in the upper half have by trend lower barriers than motors 4-9, fitted with six-membered rings in the upper half. This difference arises from the smaller five-membered ring imposing less steric hindrance in the fjord region of the motor, lowering the energy barrier required for the upper and lower halves to slide past each other during the THI. ${ }^{15}$ There is no significant effect of substitution in the $R^{1}, R^{2}$, or $R^{3}$ positions on the thermal barriers. Collectively, the THI barrier of

Table 1 DFT calculated barriers for the THI and calculated absorption maxima of the $E_{S}$ and $Z_{M}$ isomers of motors 1-9 in DMSO

\begin{tabular}{lllll}
\hline Motor & $\begin{array}{l}\boldsymbol{Z}_{\mathbf{M}} \rightarrow \boldsymbol{Z}_{\mathbf{S}} \Delta G_{\mathrm{calc}}^{\ddagger} \\
\mathrm{kcal} \mathrm{mol}\end{array}$ & $\begin{array}{l}\boldsymbol{E}_{\mathbf{M}} \rightarrow \boldsymbol{E}_{\mathbf{S}} \Delta G_{\mathrm{calc}}^{\ddagger} \\
\mathrm{kcal} \mathrm{mol}\end{array}$ & $\begin{array}{l}\boldsymbol{E}_{\mathbf{S}} \text { calc. } \\
\lambda_{\text {max }} / \mathrm{nm}\end{array}$ & $\begin{array}{c}\boldsymbol{Z}_{\mathbf{M}} \text { calc. } \\
\lambda_{\text {max }} / \mathrm{nm}\end{array}$ \\
\hline $\mathbf{1}$ & 12.1 & 16.7 & 412 & 451 \\
$\mathbf{2}$ & 12.2 & 16.6 & 415 & 451 \\
$\mathbf{3}$ & 12.9 & 16.9 & 452 & 490 \\
$\mathbf{4}$ & 27.2 & 30.6 & 388 & 440 \\
$\mathbf{5}$ & 27.7 & 29.0 & 401 & 449 \\
$\mathbf{6}$ & 26.2 & 30.0 & 406 & 457 \\
$\mathbf{7}$ & 27.1 & 30.9 & 396 & 445 \\
$\mathbf{8}$ & 27.2 & 30.8 & 395 & 444 \\
$\mathbf{9}$ & 27.3 & 30.3 & 396 & 447
\end{tabular}




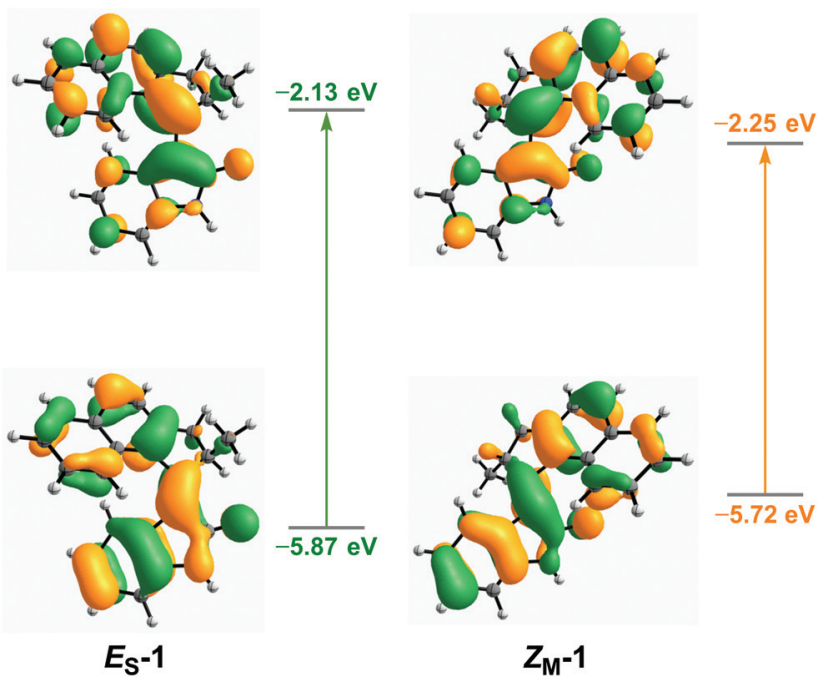

Fig. 1 Optimised structures and Frontier orbitals of $E_{\mathrm{S}}-1$ and $Z_{\mathrm{M}}-1$. Calculated with DFT at the PBE0/def2-TZVP level with the SMD DMSO solvent model.

the $Z_{\mathbf{M}} \rightarrow Z_{\mathrm{S}}$ transition is lower than that of the $\boldsymbol{E}_{\mathbf{M}} \rightarrow \boldsymbol{E}_{\mathbf{S}}$ transition because in the first case the upper half needs to slide over the carbonyl group of the oxindole lower half, which is less sterically demanding than the phenyl ring on the opposite side. This result is in agreement with previously reported barriers for oxindole motors. ${ }^{40,41}$ The computed thermochemistry suggests that motors 1-9 will follow the unidirectional rotation cycle as is typical for oxindole motors and other overcrowded alkene-based motors. ${ }^{15,40,41}$ The simulated UV-Vis spectra (see Table 1, ESI and Table S15 $\dagger$ ) for the stable states of motors 1-9 in all solvents (DMSO, $\mathrm{CH}_{2} \mathrm{Cl}_{2}$ and $\mathrm{MeOH}$ ) exhibit broad bands with absorption maxima centred between $370-450 \mathrm{~nm}$, extending further into the visible region (up to $>600 \mathrm{~nm}$ in the case of $\boldsymbol{E}_{\mathrm{S}}-3$ ). For the corresponding metastable states, the absorption maxima are red-shifted by around $30-50 \mathrm{~nm}$, due to both a destabilisation of the HOMOs of the metastable states in comparison to the HOMOs of the stable states, and a stabilisation of the LUMOs of the metastable states in comparison to that of the stable states (Fig. 1 and ESI $)$ ). In general, motors with five-membered rings in their upper halves (1-3) have absorption maxima at longer wavelengths in comparison to motors with six-membered upper halves (4-9) - owing to the increased rigidity of the five-membered ring, which improves conjugation. Indeed, this is supported by motor 1 having a smaller HOMO-LUMO gap than motors 4 and 7 (see ESI†). Functionalisation of the motors either at the upper half or at the oxindole lower half with $\mathrm{OMe}$ or $\mathrm{CN}$ groups causes a substantial red-shift (up to $40 \mathrm{~nm}$ ), in comparison to unsubstituted parent motors 1 and 4. N-Arylated motors 7-9 have similar absorption bands, showing almost no effect from functionalisation with either an electron-withdrawing or -donating moiety; presumably, this result is due to the ability of the $\mathrm{N}$-aryl bond to rotate freely, which can disrupt any potential conjugation with the central double bond axle. ${ }^{45}$

\section{Synthesis}

Motors 1-9 were synthesised in a one-pot Knoevenagel condensation of the corresponding substituted oxindoles with their upper half ketone counterparts, mediated by $\mathrm{TiCl}_{4}$ as a Lewis acid and $N$-diisopropylethylamine (DIPEA) as a base. In each case, the motor was isolated exclusively as the stable $E$ isomer, $\left(\boldsymbol{E}_{\mathbf{S}}\right.$ ), in yields varying between $34-73 \%$ (Scheme 2). The ketone derivatives were synthesised in one to four steps following well-established literature procedures. ${ }^{30,46,47}$ For motors 1-4, commercially available oxindole was used in the condensation reaction. For motors $\mathbf{5}$ and $\mathbf{6}$, the Knoevenagel condensation was carried out with commercially available 6-bromo-oxindole, yielding the bromo-functionalised motor; subsequently the aryl bromide could be subjected to either a modified Ullman etherification (yielding motor 5) or a Pd-catalysed cyanation (yielding motor 6). To yield motor 3, a Pd-catalysed cyanation was carried out on the corresponding bromo-functionalised motor. The lower halves which were later used in the Knoevenagel condensation for motors 7-9 were synthesised in a single step $\mathrm{Cu}$ catalysed $N$-arylation facilitated by $N, N^{\prime}$-dimethylethylenediamine (DMEDA), following the procedure of Buchwald and co-workers. ${ }^{48}$

Solutions of the $\boldsymbol{E}_{\mathbf{S}}$ compounds in EtOAc $(\sim 1 \mathrm{mM})$ were irradiated with $365 \mathrm{~nm}$ light until a photostationary state (PSS) was reached, to obtain the $Z$ isomers of motors 1-9 (see ESI $\dagger$ ). For motors $1-3$, the $Z_{\mathrm{S}}$ isomer was readily formed after irradiation and could be isolated by flash column chromatography (motor 1), or preparative HPLC (motor 3, see ESI $\dagger$ ). Due to poor solubility of motor 2 , bulk irradiation and isolation of the $Z_{\mathrm{S}}$ isomer was not possible. As a result of the higher activation barriers for the THI step in motors $\mathbf{4 - 9}$, as predicted by our preliminary calculations, irradiation formed the $Z_{\mathbf{M}}$ isomer. Subsequent heating of the samples in EtOAc to reflux for $2 \mathrm{~h}$ in the dark afforded the $Z_{\mathrm{S}}$ isomers, which could be subsequently isolated by flash column chromatography (for motors 4 and 7) or preparative HPLC (for motors 5, 6, and 9). For motor 8 , the $Z_{\mathrm{s}}$ isomer could not be obtained in pure form due to degradation after the prolonged heating required for the THI step to occur.

Single crystals of CN substituted motors $\boldsymbol{E}_{\mathrm{S}^{-}} \mathbf{3}, \boldsymbol{E}_{\mathrm{S}^{-}} \mathbf{6}$ and $\boldsymbol{E}_{\mathrm{S}^{-}} \mathbf{- 9}$ suitable for X-ray diffraction were grown via slow evaporation of $\mathrm{MeOH}$ into a saturated solution of the corresponding motor in $\mathrm{CHCl}_{3}$ (see Fig. 2 and ESI $\dagger$ ). The structures obtained have $\mathrm{C}(\mathrm{O})-\mathrm{N}$ and $\mathrm{C}=\mathrm{C}$ bond lengths typical for oxindole motor compounds. $^{41}$ The X-ray structures were compared with DFT optimised structures (Table 2). A root mean squares deviation

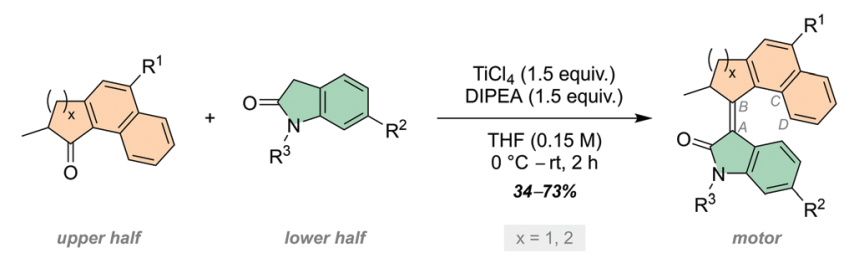

Scheme 2 General procedure for the Knoevenagel reaction in the synthesis of oxindole-based motors. 

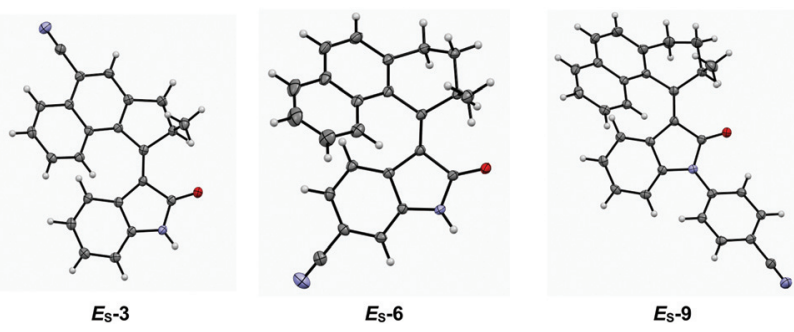

Fig. 2 ORTEP images (ellipsoid probability at 50\%) of X-ray crystal structures of motors $E_{\mathrm{s}}-3, E_{\mathrm{S}}-6$ and $E_{\mathrm{S}}-9$.

Table 2 Experimental X-ray diffraction angles and distances of $E_{\mathrm{s}}-3$, $E_{\mathrm{S}}-6$ and $E_{\mathrm{S}}-9$

\begin{tabular}{|c|c|c|c|c|}
\hline Motor & $\begin{array}{l}\mathrm{C}(\mathrm{O})-\mathrm{N} \\
\text { distance/Å }\end{array}$ & $\begin{array}{l}\mathrm{C}=\mathrm{C} \\
\text { distance } / \AA\end{array}$ & $\begin{array}{l}\text { Dihedral } \\
\text { angle }^{a} / o\end{array}$ & $\begin{array}{l}\mathrm{RMSD} / \\
\AA \\
\AA\end{array}$ \\
\hline$E_{\mathrm{S}}-3$ & $1.360(1.381)$ & $1.360(1.358)$ & $33.07(35.24)$ & 0.0869 \\
\hline$E_{\mathrm{S}}-6$ & $1.368(1.384)$ & $1.363(1.360)$ & $53.91(47.41)$ & 0.306 \\
\hline$E_{\mathrm{S}^{-9}}$ & $1.403(1.402)$ & $1.358(1.360)$ & $47.60(47.33)$ & 0.264 \\
\hline
\end{tabular}

Values of the computationally optimised structures are shown in brackets. ${ }^{a}$ Dihedral $=$ ABCD in Scheme 2 .

was performed to compare the X-ray structures with the DFT simulated structures, in all cases the value was under $0.4 \AA$. $\boldsymbol{E}_{\mathrm{S}^{-}}$ 3 has a lower value due to the rigidity of the five-membered ring in the upper half, which may also contribute to the increased conjugation and red-shift observed for motors 1-3. Conversely, $\boldsymbol{E}_{\mathbf{S}} \mathbf{- 6}$ and $\boldsymbol{E}_{\mathbf{S}} \mathbf{- 9}$ possess six-membered rings in their upper halves which can pucker, resulting in multiple possible conformers; this is where the deviation in the RMSD originates from (see ESI, Fig. S5 $\dagger$ ).

The calculated and experimental values for $\mathrm{C}(\mathrm{O})-\mathrm{N}$ and $\mathrm{C}=\mathrm{C}$ bond lengths of all the motors are in good agreement. For $\boldsymbol{E}_{\mathbf{S}^{-6}} \mathbf{6}$, the dihedral angle in the X-ray structure is $5^{\circ}$ larger than in the computed structure, which is possibly due to packing effects in the crystalline solid. It would be expected that the dihedral angle in solution would be more similar to its computationally simulated value and to the values observed for $\boldsymbol{E}_{\mathbf{S}} \mathbf{- 9}$, as the two compounds have similar steric demand in the fjord region.

\section{Steady state spectroscopy}

With motors 1-9 in hand, the photochemical behaviour was first studied by UV-Vis spectroscopy. UV-Vis measurements were performed on the $\boldsymbol{E}_{\mathbf{S}}$ isomers of each motor in $\mathrm{CH}_{2} \mathrm{Cl}_{2}$, DMSO and MeOH (see ESI, Fig. S20-S28†). All the motors show a broad absorption band with an absorption maximum between 350-400 $\mathrm{nm}$, further tailing into the visible region (upwards of $500 \mathrm{~nm}$ ). The simulated UV-vis spectra are in good agreement with the experimentally obtained spectra. Indeed, as predicted by DFT calculations, functionalisation of the motors with either OMe or CN groups induces a red-shift of the absorption maxima of the $\pi-\pi^{*}$ band by up to $40 \mathrm{~nm}$. We observed very little solvatochromism between the three solvents investigated $(<5 \mathrm{~nm})$ for all of the motors. Due to poor solubility in $\mathrm{CH}_{2} \mathrm{Cl}_{2}$ and $\mathrm{MeOH}$, UV-Vis measurements of motor 2 were only carried out in DMSO and THF. Motor 2 demonstrates a small degree of solvatochromism with the absorption band in DMSO being red-shifted by $7 \mathrm{~nm}$ compared to THF (Fig. S21†).

Upon irradiation of a sample of the stable $E$ isomer of motor $4\left(\boldsymbol{E}_{\mathbf{S}^{-4}}\right)$ with a $365 \mathrm{~nm}$ LED in DMSO at room temperature, a bathochromic shift occurs with a clear isosbestic point (Fig. 3A). We assign this observation to the formation of the metastable $Z$ isomer, $\boldsymbol{Z}_{\mathbf{M}} \mathbf{- 4}$. Subsequent heating of the sample to $60{ }^{\circ} \mathrm{C}$ forms the stable $Z$ isomer, $Z_{\mathbf{S}^{-4}}$, accompanied by a hypsochromic shift in the absorption maximum. Further irradiation of the sample induces a second bathochromic shift, signifying formation of the metastable $E$ isomer, $\boldsymbol{E}_{\mathbf{M}^{-4}}$, and finally upon heating to $100{ }^{\circ} \mathrm{C}$, a second hypsochromic shift indicates the formation of the stable $E$ isomer $\left(\boldsymbol{E}_{\mathbf{S}}-\mathbf{4}\right)$ once again, completing the $360^{\circ}$ rotation of the motor. The same experiments were carried out for motors 5-9 indicating that all of the compounds act as unidirectional motors (see ESI, Fig. S32-S37†).

Due to the low thermal barriers for the THI conversion in oxindole motors with upper halves containing five-membered rings, ${ }^{40,41}$ the metastable states of motors 1-3 were not observable with UV-Vis while irradiating at room temperature. When irradiating the $\boldsymbol{E}_{\mathbf{S}}$ isomer with $365 \mathrm{~nm}$ light, we recorded a small bathochromic shift of $\boldsymbol{E}_{\mathbf{S}}$ 1-3 upon irradiation. This observation indicates formation of the $Z_{\mathbf{S}}$ isomer at the PSS. The $Z_{\mathbf{S}}$ form of the motors is slightly red-shifted in comparison to the $\boldsymbol{E}_{\mathbf{S}}$ isomer, according to simulated absorption spectra (see ESI, Fig. S29 and S31 $\dagger$ ) and our previous studies. ${ }^{40,41}$ Irradiation of motor 2 in $\operatorname{THF}(\lambda=365 \mathrm{~nm})$ also showed a small bathochromic shift while reaching the PSS (see ESI, Fig. S30†).

Next, the photochemical and thermal isomerisation behaviour of motors 1-9 were investigated by ${ }^{1} \mathrm{H}$ NMR spectroscopy (Fig. 3B and ESI, Fig. S14 $\dagger$ ). Upon irradiation of a sample of $\boldsymbol{E}_{\mathbf{S}} \mathbf{- 4}$ in DMSO- $d_{6}$ with $395 \mathrm{~nm}$ light at room temperature, a new set of signals appeared. A substantial shift of the protons of the methyl group $\left(\mathrm{H}_{\mathrm{a}}\right)$ and of the allylic proton $\left(\mathrm{H}_{\mathrm{b}}\right)$ was observed (see Fig. 3A for atom labelling), allowing identification of the newly formed metastable $Z$ isomer, $Z_{\mathbf{M}} \mathbf{- 4}$, in the photochemical $E-Z$ isomerisation (PEZ) step. The sample was irradiated until no further spectral changes were observed, yielding a PSS with a 52:48 $\boldsymbol{E}_{\mathbf{S}} \mathbf{- 4}: \boldsymbol{Z}_{\mathbf{M}^{-4}}$ distribution. The sample was then heated to $70{ }^{\circ} \mathrm{C}$, resulting in the disappearance of the peaks corresponding to the $\boldsymbol{Z}_{\mathbf{M}} \mathbf{- 4}$ isomer and an appearance of a new set of peaks, indicating the formation of the stable $Z$ isomer $\left(\boldsymbol{Z}_{\mathbf{S}} \mathbf{- 4}\right)$ following the exergonic thermal helix inversion (THI) step. The NMR sample was irradiated once again with $395 \mathrm{~nm}$ light and a fourth state was revealed, corresponding to formation of the metastable $E$ isomer, $\boldsymbol{E}_{\mathbf{M}^{-4}}$, with nearly quantitative conversion of $Z_{\mathbf{S}^{-4}} \rightarrow \boldsymbol{E}_{\mathbf{M}^{\mathbf{- 4}}}\left(\mathrm{PSS}_{395}=\right.$ $4: 96, Z_{\mathbf{S}} \mathbf{- 4}: \boldsymbol{E}_{\mathbf{M}^{-4}}$ ). Upon heating to $100{ }^{\circ} \mathrm{C}$, the initial isomer $\boldsymbol{E}_{\mathrm{S}} \mathbf{- 4}$ was reformed, signifying the second THI step of the 
(A)
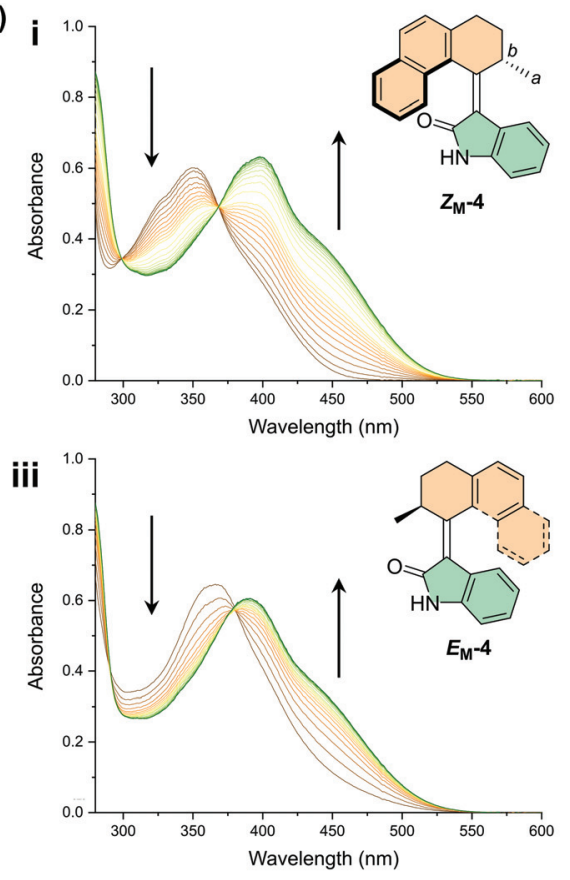

(B)
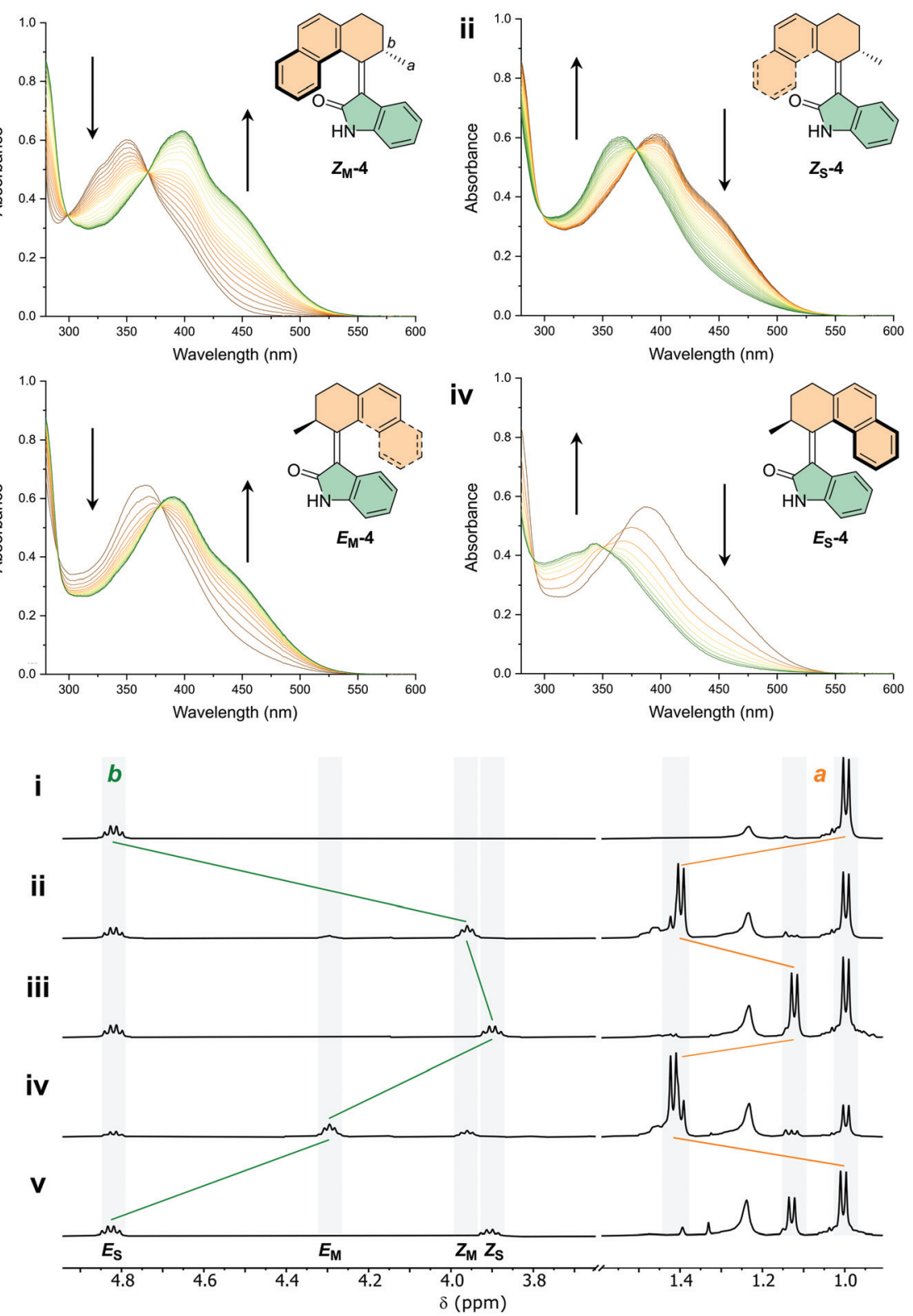

Fig. 3 (A) UV-Vis irradiation cycle of 4 in DMSO ( $c=\sim 2 \times 10^{-5} \mathrm{M}$ ). (i) irradiation to PSS with $\lambda=365 \mathrm{~nm}$; (ii) heating to $60^{\circ} \mathrm{C}$, THI; (iii) irradiation to PSS with $\lambda=365 \mathrm{~nm}$; (iv) heating to $100{ }^{\circ} \mathrm{C}$, THI. (B) ${ }^{1} \mathrm{H}$ NMR irradiation studies of 4 in DMSO- $d_{6}\left(c=2.4 \times 10^{-3} \mathrm{M}\right.$ ). (i) $E_{\mathrm{S}}-4$ before irradiation; (ii) $\mathrm{PSS}_{395}, 52$ : $48\left(E_{\mathrm{S}}-4: Z_{\mathrm{M}}-4\right)$; (iii) $\mathrm{THI}, 70^{\circ} \mathrm{C}, 60 \mathrm{~min}$; (iv) $\mathrm{PSS}_{395}, 4: 96\left(Z_{\mathrm{S}}-4: E_{\mathrm{M}}-4\right)$; (v) $\mathrm{THI}, 100{ }^{\circ} \mathrm{C}, 180 \mathrm{~min}$.

rotation cycle and a full $360^{\circ}$ unidirectional rotation. Similar rotation cycles were observed for motors 5-9 at irradiation wavelengths ranging from between $395-420 \mathrm{~nm}$, depending on the absorption band of the motor (see ESI, Fig. S14-S19†).

Owing to the low activation barriers for the THI steps in motors 1-3, it was necessary to carry out low temperature NMR experiments at $-90{ }^{\circ} \mathrm{C}$ in $\mathrm{CD}_{2} \mathrm{Cl}_{2}$ to observe the metastable states. Under constant irradiation of the $\boldsymbol{E}_{\mathbf{S}}$ isomer, the first new set of peaks that arise correspond to the $\boldsymbol{Z}_{\mathbf{M}}$ isomer. The calculated barrier for the $Z_{\mathbf{M}} \rightarrow Z_{\mathrm{S}}$ transition in motors 1-3 is $\sim 12 \mathrm{kcal} \mathrm{mol}^{-1}$, a barrier which is so low that even at $-90{ }^{\circ} \mathrm{C}$ the $Z_{\mathbf{M}}$ isomer was observed to undergo THI to form the $Z_{\mathbf{S}}$

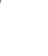


Additional NMR irradiation experiments were carried out in DMSO for motors 1-9 at wavelengths close to the end of their respective $\pi \rightarrow \pi^{*}$ absorption bands, to assess motor operation at longer wavelengths. The PSS ratios at these wavelengths are presented in Table 3. For motors 1-3, $505 \mathrm{~nm}$ light was used, improving the PSS ratios for these motors in comparison to irradiation at $365 \mathrm{~nm}$. Motors 4-6 were irradiated with $455 \mathrm{~nm}$ light, and 7-9 with $420 \mathrm{~nm}$ light. Although with lower PSS ratios than when irradiating near the absorption maximum (ca. $365 \mathrm{~nm}$ ), motors $\mathbf{4 - 9}$ operate under visible light illumination.

Eyring analysis was performed to determine the activation parameters for the THI steps motors 1-9, and the data is compiled in Table 4. For motors 4-9, the rates of the THI steps were determined by following the decay in absorbance of the absorption band of the metastable state in toluene $(\lambda=$ 400-450 nm, see ESI $\dagger$ ). For motors 1 and 3, low temperature ${ }^{1} \mathrm{H}$ NMR in $d_{4}$-methanol was employed to follow the rate of conversion of the metastable states to their corresponding stable states (see ESI $\dagger$ ). The rate of THI was followed at five

Table 3 PSSs for motors 1-9

\begin{tabular}{lll}
\hline Motor & $\operatorname{PSS}_{365}\left(\boldsymbol{E}_{\mathbf{S}}: Z_{\mathbf{S}}\right)$ & $\operatorname{PSS}_{505}\left(\boldsymbol{E}_{\mathbf{S}}: Z_{\mathbf{S}}\right)$ \\
\hline $\mathbf{1}$ & $75: 25$ & $59: 41$ \\
$\mathbf{2}$ & $64: 36$ & $61: 49$ \\
$\mathbf{3}$ & $69: 31$ & $44: 56$ \\
\hline
\end{tabular}

\begin{tabular}{|c|c|c|c|c|}
\hline \multirow[b]{2}{*}{ Motor } & \multicolumn{2}{|l|}{$\mathrm{PSS}_{365}$} & \multicolumn{2}{|l|}{$\mathrm{PSS}_{\text {vis }}$} \\
\hline & $\left(E_{\mathbf{S}}: Z_{\mathbf{M}}\right)$ & $\left(Z_{\mathbf{S}}: E_{\mathbf{M}}\right)$ & $\left(E_{\mathbf{S}}: Z_{\mathbf{M}}\right)$ & $\left(Z_{\mathrm{S}}: E_{M}\right)$ \\
\hline 4 & $17: 83$ & $12: 88$ & $84: 16^{a}$ & $32: 68^{a}$ \\
\hline 5 & $23: 77$ & $10: 90$ & $80: 20^{a}$ & $21: 79^{a}$ \\
\hline 6 & $12: 88$ & $10: 90$ & $72: 28^{a}$ & $39: 61^{a}$ \\
\hline 7 & $16: 84$ & $5: 95$ & $67: 33^{b}$ & $9: 91^{b}$ \\
\hline 8 & $17: 83$ & $7: 92$ & $72: 28^{b}$ & $12: 88^{b}$ \\
\hline 9 & $18: 82$ & $2: 98$ & $71: 29^{b}$ & $13: 87^{b}$ \\
\hline
\end{tabular}

Table 4 Experimentally determined thermal barriers for the THI steps of motors 1, 3-9

\begin{tabular}{lll}
\hline Motor & $Z_{\mathbf{M}} \rightarrow Z_{\mathbf{S}} \Delta G^{\ddagger} / \mathrm{kcal} \mathrm{mol}^{-1}$ & $\boldsymbol{E}_{\mathbf{M}} \rightarrow \boldsymbol{E}_{\mathbf{S}} \Delta G^{\ddagger} / \mathrm{kcal} \mathrm{mol}^{-1}$ \\
\hline $\mathbf{1}$ & $-^{a}$ & $17 \pm 1$ \\
$\mathbf{3}$ & $-^{a}$ & $16 \pm 1$ \\
$\mathbf{4}$ & $23.5 \pm 0.3$ & $25.8 \pm 0.5$ \\
$\mathbf{5}$ & $23.3 \pm 0.2$ & $26.4 \pm 0.2$ \\
$\mathbf{6}$ & $23.2 \pm 0.6$ & $24.0 \pm 0.5$ \\
$\mathbf{7}$ & $23.6 \pm 0.4$ & $25.5 \pm 0.7$ \\
$\mathbf{8}$ & $23.9 \pm 0.1$ & $-{ }^{b}$ \\
$\mathbf{9}$ & $23.9 \pm 0.4$ & $26.0 \pm 0.1$
\end{tabular}

Barriers for motors 1 and 3 determined by low temperature NMR in $d_{4}$ methanol. Barriers for motors 4-9 determined by UV-Vis spectroscopy in toluene. ${ }^{a} \boldsymbol{Z}_{\mathbf{M}}$ isomers could not be sufficiently populated at $-90{ }^{\circ} \mathrm{C}$ to monitor conversion to the $Z_{\mathrm{S}}$ state. ${ }^{b} Z_{\mathrm{S}}$ could not be obtained in pure form due to degradation after prolonged heating required for the THI step to occur. temperatures in each case, after which the rate constants obtained for the decay at each temperature were used to calculate the activation parameters using the linearised Eyring equation (see ESI $\dagger$ ). All experimentally measured barriers follow qualitatively the same trend as calculated by DFT. As anticipated from calculations and previous studies on oxindole motors, ${ }^{40,41}$ the $\boldsymbol{E}_{\mathbf{M}} \rightarrow \boldsymbol{E}_{\mathbf{S}}$ transition is the rate-determining step in the rotational cycle.

Motors 4-9 follow a clear trend for both THI steps, with small fluctuations in the barriers between each compound $\left(<2 \mathrm{kcal} \mathrm{mol} \mathrm{m}^{-1}\right)$. The thermal half-lives of the barriers at $293.15 \mathrm{~K}$ equate to multiple hours $(6-20 \mathrm{~h})$ for the $Z_{\mathbf{M}} \rightarrow Z_{\mathbf{S}}$ transitions, and multiple days (13-64 d) for the $\boldsymbol{E}_{\mathbf{M}} \rightarrow \boldsymbol{E}_{\mathbf{S}}$ transitions. The single outlier is the barrier from $\boldsymbol{E}_{\mathbf{M}^{\mathbf{- 6}}} \rightarrow \boldsymbol{E}_{\mathbf{S}} \mathbf{- 6}$, which has a slightly shorter half-life at $293.15 \mathrm{~K}$ of $27 \mathrm{~h}$, which interestingly coincides with the larger dihedral angle found in the crystal structure of $\boldsymbol{E}_{\mathbf{S}} \mathbf{- 6}$. Overall, this trend demonstrates that substitution of electron-withdrawing or -donating groups at the 5-position or installing aryl groups on the amide functionality of oxindole has little to no effect on the THI barriers for these compounds.

Motors 1 and 3 have considerably lower THI barriers than for motors 4-9 because of the decreased degree of steric demand in the fjord region of these motors. The experimental barriers for these motors for the $\boldsymbol{E}_{\mathbf{M}} \rightarrow \boldsymbol{E}_{\mathbf{S}}$ THI steps are around $17 \mathrm{kcal} \mathrm{mol}^{-1}$, a result that is in agreement with the calculated thermal barriers and that is also similar to the experimentally obtained barriers of structurally related oxindole motors. ${ }^{40,41}$ Calculated barriers for the $\boldsymbol{Z}_{\mathbf{M}} \rightarrow \boldsymbol{Z}_{\mathbf{S}}$ barriers are approximately $12 \mathrm{kcal} \mathrm{mol}^{-1}$; due to these low activation energies, experimental derivation of the barriers was challenging. Even at $-90{ }^{\circ} \mathrm{C}$, the $\boldsymbol{Z}_{\mathbf{M}}$ states could not be sufficiently populated to follow conversion to the corresponding stable $Z_{\mathbf{S}}$ state. As the rate limiting step of the rotation cycle is indeed the $\boldsymbol{E}_{\mathbf{M}} \rightarrow \boldsymbol{E}_{\mathbf{S}}$ THI step, the half-lives of motors $\mathbf{1}$ and $\mathbf{3}$ are found to be on the scale of milliseconds at $293.15 \mathrm{~K}$.

The quantum yields (QYs) of motors 1-9 were determined using the method of Stranius and Börjesson, ${ }^{49}$ following the evolution of both components of the photostationary distribution upon irradiation $(\lambda=365 \mathrm{~nm})$ of a sample of known concentration in UV-Vis at room temperature. In the case of motors 1-3, the concentration of the metastable states at room temperature is negligible. Therefore the measured QY is approximated as a forwards $\boldsymbol{E}_{\mathbf{S}} \rightarrow \boldsymbol{Z}_{\mathbf{S}}$ transition and a backwards $\boldsymbol{Z}_{\mathbf{S}} \rightarrow \boldsymbol{E}_{\mathbf{S}}$ transition, neglecting the contribution of the metastable states. ${ }^{41}$ Due to their longer thermal half-lives, the forwards and backwards photoreactions for all states in the rotation cycle of motors 4-9 can be measured. The QYs of motors 1-9 are shown in Table 5, which are generally higher than previously reported oxindole motors. ${ }^{40,41}$ In all cases, the photoreactions of the $Z_{\mathrm{S}}$ isomers are more efficient than for the $\boldsymbol{E}_{\mathbf{S}}$ isomers. It has to be noted that the introduction of a free amide functionality in motor $\mathbf{4}$ doubles the QY for the $\boldsymbol{E}_{\mathbf{S}}$ $\rightarrow Z_{\mathbf{M}}$ step (from $2.3 \%-6.7 \%$ ) and increases six-fold for the $Z_{\mathbf{S}}$ $\rightarrow \boldsymbol{E}_{\mathbf{M}}$ step (from $2.1 \%-12.9 \%$ ), in comparison to the previously reported parent motor - fitted with an $N$-Me group at 
Table 5 Quantum yields of motors 1-9

\begin{tabular}{lllllll}
\hline \multicolumn{7}{c}{ Quantum yield, $\varphi(\%)$} \\
\cline { 2 - 7 } Motor & $\boldsymbol{E}_{\mathbf{S}} \rightarrow Z_{\mathbf{S}}$ & $\boldsymbol{E}_{\mathbf{S}} \rightarrow Z_{\mathbf{M}}$ & $Z_{\mathbf{M}} \rightarrow \boldsymbol{E}_{\mathbf{S}}$ & $\boldsymbol{Z}_{\mathbf{S}} \rightarrow \boldsymbol{E}_{\mathbf{S}}$ & $Z_{\mathbf{S}} \rightarrow \boldsymbol{E}_{\mathbf{M}}$ & $\boldsymbol{E}_{\mathbf{M}} \rightarrow Z_{\mathbf{S}}$ \\
\hline $\mathbf{1}$ & 6.7 & - & - & 16.9 & - & - \\
$\mathbf{2}$ & 10.1 & - & - & 14.9 & - & - \\
$\mathbf{3}$ & 10.8 & - & - & 22.5 & - & - \\
$\mathbf{4}$ & - & 6.7 & 1.6 & - & 12.9 & 3.1 \\
$\mathbf{5}$ & - & 5.3 & 1.8 & - & 11.4 & 1.6 \\
$\mathbf{6}$ & - & 7.3 & 1.7 & - & 18.2 & 3.4 \\
$\mathbf{7}$ & - & 3.9 & 1.2 & - & 17.8 & 1.4 \\
$\mathbf{8}$ & - & 3.2 & 1.0 & - & - & - \\
$\mathbf{9}$ & - & 3.2 & 1.4 & - & 16.1 & 0.9
\end{tabular}

the amide. ${ }^{40} \mathrm{~N}$-Arylated motors 7-9 do not possess much higher QYs for the $\boldsymbol{E}_{\mathbf{S}} \rightarrow \boldsymbol{Z}_{\mathbf{M}}$ step than previously reported structurally similar oxindole motors, ${ }^{40}$ however the values for the $\boldsymbol{Z}_{\mathbf{S}} \rightarrow \boldsymbol{E}_{\mathbf{M}}$ step increases up to nine-fold. Interestingly, the $\mathrm{CN}$ functionalised motors have higher QYs than their unsubstituted counterparts and OMe functionalised motors have lower QYs than their unsubstituted counterparts. Such trends were also found for fluorene-based molecular motors, ${ }^{42}$ and confirms the computational prediction that having electron withdrawing groups in conjugation with the central alkene bond increases the isomerisation quantum yields. ${ }^{50}$

\section{Conclusion}

In summary, we presented the facile functionalisation of molecular motors based on oxindole at three different positions along the motor skeleton, with either $\mathrm{CN}$ or OMe groups. The typical four-step rotation cycle of oxindole-based molecular motors were observed for the motors newly prepared in this work, and the desirable properties of the molecular motors have been fully retained i.e. they are readily synthesised and visible-light addressable. Functionalisation on either the upper or lower half with OMe or CN groups conjugated directly into the double bond axle of the motors further red-shifts the absorption wavelength more towards the visible region of the electromagnetic spectrum, however substitutions in these positions has little to no effect on the rotational speed of these motors. Therefore, functionalisation of the motors in these positions is possible, whilst keeping the rotational speed and motor functionality consistent; a useful feature for further application of the motors. All of the new motors presented can be operated under visible light illumination; for motors 1-3 the PSS ratios are even improved. The QYs of the motors are considerably higher than those previously reported, showing that further functionalisation of the oxindole motor scaffold (either with aryl functionalities on the amide, or electron-withdrawing or -donating groups on the backbone) can tune the quantum efficiency of these motors. This work further solidifies oxindole-based motors as visible-light responsive systems to induce controlled motion, viable for future applications in biological and materials science applications.

\section{Author contributions}

B. L. F. designed the study. D. R. S. P. synthesised and characterised the compounds investigated, performed preparative HPLC, NMR and UV-Vis measurements, and X-ray diffraction measurements. D. R. S. P. and S. C. measured and determined the quantum yields. D. D. performed DFT calculations. B. L. F. and S. C. supervised the work. D. R. S. P. and S. C. wrote the paper. All authors discussed and commented on the manuscript. S. C. and B. L. F. acquired funding.

\section{Conflicts of interest}

The authors declare there to be no conflicts of interest.

\section{Acknowledgements}

D. R. S. P. gratefully acknowledges Pieter van der Meulen and Dr Johan Kemmink for guidance with low temperature NMR measurements and unlimited access to their liquid $\mathrm{N}_{2}$ Dewar. She acknowledges Renze Sneep for HRMS measurements, in addition to HPLC maintenance and guidance with preparative HPLC; she also acknowledges Dr Romain Costil for guidance with preparative HPLC and fruitful discussions. She gratefully acknowledges Dr Ryojun Toyoda for guidance in measuring and solving the X-ray structures. D. R. S. P. acknowledges Dr Niklas Thiel, Dr Wojciech Danowski and Cosima Stähler for fruitful discussions. We thank the Centre for Information Technology of the University of Groningen for their support and for providing access to the Peregrine high performance computing cluster. Financial support from the Horizon 2020 Framework Programme (ERC Advanced Investigator Grant No. 694345 to B. L. F.), the Netherlands Ministry of Education, Culture and Science (Gravitation Programme 024.001.035 to B. L. F.) and the Marie Skłodowska-Curie Action (Individual Fellowship No. 838280 to S. C.) is gratefully acknowledged.

\section{References}

1 J. P. Sauvage, From Chemical Topology to Molecular Machines (Nobel Lecture), Angew. Chem., Int. Ed., 2017, 56, 11080-11093.

2 J. F. Stoddart, Mechanically Interlocked Molecules (MIMs) -Molecular Shuttles, Switches, and Machines (Nobel Lecture), Angew. Chem., Int. Ed., 2017, 56, 11094-11125.

3 B. L. Feringa, The Art of Building Small: From Molecular Switches to Motors (Nobel Lecture), Angew. Chem., Int. Ed., 2017, 56, 11060-11078.

4 W. R. Browne and B. L. Feringa, Molecular Switches, WileyVCH Verlag GmbH \& Co. KGaA, Weinheim, Germany, 2011.

5 M. Baroncini, S. Silvi and A. Credi, Photo- and RedoxDriven Artificial Molecular Motors, Chem. Rev., 2020, 120, 200-268. 
6 D. Dattler, G. Fuks, J. Heiser, E. Moulin, A. Perrot, X. Yao and N. Giuseppone, Design of Collective Motions from Synthetic Molecular Switches, Rotors, and Motors, Chem. Rev., 2020, 120, 310-433.

7 V. García-López, D. Liu and J. M. Tour, Light-Activated Organic Molecular Motors and Their Applications, Chem. Rev., 2020, 120, 79-124.

8 R. D. Astumian, How molecular motors work - insights from the molecular machinist's toolbox: the Nobel prize in Chemistry 2016, Chem. Sci., 2017, 8, 840-845.

9 Y. Feng, M. Ovalle, J. S. W. Seale, C. K. Lee, D. J. Kim, R. D. Astumian and J. F. Stoddart, Molecular Pumps and Motors, J. Am. Chem. Soc., 2021, 143, 5569-5591.

10 I. Aprahamian, The Future of Molecular Machines, ACS Cent. Sci., 2020, 6, 347-358.

11 A. W. Heard and S. M. Goldup, Simplicity in the Design, Operation, and Applications of Mechanically Interlocked Molecular Machines, ACS Cent. Sci., 2020, 6, 117-128.

12 N. Giuseppone and A. Walther, Out-of-Equilibrium (Supra) molecular Systems and Materials, Wiley, Hoboken, NJ, 1st edn, 2021.

13 N. Koumura, R. W. J. Zijlstra, R. A. van Delden, N. Harada and B. L. Feringa, Light-driven monodirectional molecular rotor, Nature, 1999, 401, 152-155.

14 S. Kassem, T. van Leeuwen, A. S. Lubbe, M. R. Wilson, B. L. Feringa and D. A. Leigh, Artificial molecular motors, Chem. Soc. Rev., 2017, 46, 2592-2621.

15 D. R. S. Pooler, A. S. Lubbe, S. Crespi and B. L. Feringa, Designing light-driven rotary molecular motors, Chem. Sci., 2021, 12, 14964-14986.

16 S. Krause and B. L. Feringa, Towards artificial molecular factories from framework-embedded molecular machines, Nat. Rev. Chem., 2020, 4, 550-562.

17 Q. Zhang, D. H. Qu, H. Tian and B. L. Feringa, Bottom-Up: Can Supramolecular Tools Deliver Responsiveness from Molecular Motors to Macroscopic Materials?, Matter, 2020, 3, 355-370.

18 W. Danowski, T. van Leeuwen, S. Abdolahzadeh, D. Roke, W. R. Browne, S. J. Wezenberg and B. L. Feringa, Unidirectional rotary motion in a metal-organic framework, Nat. Nanotechnol., 2019, 14, 488-494.

19 J. Vicario, A. Meetsma and B. L. Feringa, Controlling the speed of rotation in molecular motors. Dramatic acceleration of the rotary motion by structural modification, Chem. Commun., 2005, 5910-5912.

20 R. Dorel and B. L. Feringa, Stereodivergent Anion Binding Catalysis with Molecular Motors, Angew. Chem., Int. Ed., 2020, 59, 785-789.

21 K. Grill and H. Dube, Supramolecular relay-control of organocatalysis with a hemithioindigo-based molecular motor, J. Am. Chem. Soc., 2020, 142, 19300-19307.

22 V. García-López, F. Chen, L. G. Nilewski, G. Duret, A. Aliyan, A. B. Kolomeisky, J. T. Robinson, G. Wang, R. Pal and J. M. Tour, Molecular machines open cell membranes, Nature, 2017, 548, 567-572.
23 A. S. Lubbe, Q. Liu, S. J. Smith, J. W. De Vries, J. C. M. Kistemaker, A. H. De Vries, I. Faustino, Z. Meng, W. Szymanski, A. Herrmann and B. L. Feringa, Photoswitching of DNA Hybridization Using a Molecular Motor, J. Am. Chem. Soc., 2018, 140, 5069-5076.

24 Q. Zhou, J. Chen, Y. Luan, P. A. Vainikka, S. Thallmair, S. J. Marrink, B. L. Feringa and P. van Rijn, Unidirectional rotating molecular motors dynamically interact with adsorbed proteins to direct the fate of mesenchymal stem cells, Sci. Adv., 2020, 6, eaay2756.

25 K. Kinbara and T. Aida, Toward Intelligent Molecular Machines: Directed Motions of Biological and Artificial Molecules and Assemblies, Chem. Rev., 2005, 105, 13771400.

26 D. Bléger and S. Hecht, Visible-Light-Activated Molecular Switches, Angew. Chem., Int. Ed., 2015, 54, 11338-11349.

27 T. van Leeuwen, J. Pol, D. Roke, S. J. Wezenberg and B. L. Feringa, Visible-Light Excitation of a Molecular Motor with an Extended Aromatic Core, Org. Lett., 2017, 19, 14021405.

28 D. Roke, B. L. Feringa and S. J. Wezenberg, A Visible-LightDriven Molecular Motor Based on Pyrene, Helv. Chim. Acta, 2019, 102, e1800221.

29 D. Roke, C. Stuckhardt, W. Danowski, S. J. Wezenberg and B. L. Feringa, Light-Gated Rotation in a Molecular Motor Functionalized with a Dithienylethene Switch, Angew. Chem., Int. Ed., 2018, 57, 10515-10519.

30 L. Pfeifer, M. Scherübl, M. Fellert, W. Danowski, J. Cheng, J. Pol and B. L. Feringa, Photoefficient 2nd generation molecular motors responsive to visible light, Chem. Sci., 2019, 10, 8768-8773.

31 R. A. van Delden, N. Koumura, A. Schoevaars, A. Meetsma and B. L. Feringa, A donor-acceptor substituted molecular motor: unidirectional rotation driven by visible light, Org. Biomol. Chem., 2003, 1, 33-35.

32 L. Pfeifer, N. V. Hoang, M. Scherübl, M. S. Pshenichnikov and B. L. Feringa, Powering rotary molecular motors with low-intensity near-infrared light, Sci. Adv., 2020, 6, eabb6165.

33 A. Cnossen, L. Hou, M. M. Pollard, P. V. Wesenhagen, W. R. Browne and B. L. Feringa, Driving unidirectional molecular rotary motors with visible light by intra- and intermolecular energy transfer from palladium porphyrin, J. Am. Chem. Soc., 2012, 134, 17613-17619.

34 L. Greb and J. M. Lehn, Light-driven molecular motors: Imines as four-step or two-step unidirectional rotors, J. Am. Chem. Soc., 2014, 136, 13114-13117.

35 L. Greb, A. Eichhöfer and J. M. Lehn, Synthetic Molecular Motors: Thermal $\mathrm{N}$ Inversion and Directional Photoinduced $\mathrm{C}=\mathrm{N}$ Bond Rotation of Camphorquinone Imines, Angew. Chem., Int. Ed., 2015, 54, 14345-14348.

36 M. Guentner, M. Schildhauer, S. Thumser, P. Mayer, D. Stephenson, P. J. Mayer and H. Dube, Sunlight-powered $\mathrm{kHz}$ rotation of a hemithioindigo-based molecular motor, Nat. Commun., 2015, 6, 8406. 
37 L. A. Huber, K. Hoffmann, S. Thumser, N. Böcher, P. Mayer and H. Dube, Direct Observation of Hemithioindigo-Motor Unidirectionality, Angew. Chem., Int. Ed., 2017, 56, 1453614539.

38 A. Gerwien, M. Schildhauer, S. Thumser, P. Mayer and H. Dube, Direct evidence for hula twist and single-bond rotation photoproducts, Nat. Commun., 2018, 9, 2510.

39 A. Gerwien, P. Mayer and H. Dube, Photon-Only Molecular Motor with Reverse Temperature-Dependent Efficiency, J. Am. Chem. Soc., 2018, 140, 16442-16445.

40 D. Roke, M. Sen, W. Danowski, S. J. Wezenberg and B. L. Feringa, Visible-Light-Driven Tunable Molecular Motors Based on Oxindole, J. Am. Chem. Soc., 2019, 141, 7622-7627.

41 D. R. S. Pooler, R. Pierron, S. Crespi, R. Costil, L. Pfeifer, J. Léonard, M. Olivucci and B. L. Feringa, Effect of chargetransfer enhancement on the efficiency and rotary mechanism of an oxindole-based molecular motor, Chem. Sci., 2021, 12, 7486-7497.

42 J. Conyard, A. Cnossen, W. R. Browne, B. L. Feringa and S. R. Meech, Chemically Optimizing Operational Efficiency of Molecular Rotary Motors, J. Am. Chem. Soc., 2014, 136, 9692-9700.

43 M. M. Pollard, P. V. Wesenhagen, D. Pijper and B. L. Feringa, On the effect of donor and acceptor substituents on the behaviour of light-driven rotary molecular motors, Org. Biomol. Chem., 2008, 6, 1605-1612.
44 A. V. Marenich, C. J. Cramer and D. G. Truhlar, Universal solvation model based on solute electron density and on a continuum model of the solvent defined by the bulk dielectric constant and atomic surface tensions, J. Phys. Chem. B, 2009, 113, 6378-6396.

45 F. L. Kiss, B. P. Corbet, N. A. Simeth, B. L. Feringa and S. Crespi, Predicting the substituent effects in the optical and electrochemical properties of $\mathrm{N}, \mathrm{N}^{\prime}$-substituted isoindigos, Photochem. Photobiol. Sci., 2021, 20, 927-938.

46 P. Štacko, J. C. M. Kistemaker and B. L. Feringa, FluorineSubstituted Molecular Motors with a Quaternary Stereogenic Center, Chem. - Eur. J., 2017, 23, 6643-6653.

47 J. C. M. Kistemaker, S. F. Pizzolato, T. van Leeuwen, T. C. Pijper and B. L. Feringa, Spectroscopic and Theoretical Identification of Two Thermal Isomerization Pathways for Bistable Chiral Overcrowded Alkenes, Chem. Eur. J., 2016, 22, 13478-13487.

48 R. A. Altman, A. M. Hyde, X. Huang and S. L. Buchwald, Orthogonal Pd- and Cu-Based Catalyst Systems for C- and N-Arylation of Oxindoles, J. Am. Chem. Soc., 2008, 130, 9613-9620.

49 K. Stranius and K. Börjesson, Determining the photoisomerization quantum yield of photoswitchable molecules in solution and in the solid state, Sci. Rep., 2017, 7, 1-9.

50 M. Filatov and M. Olivucci, Designing conical intersections for light-driven single molecule rotary motors: From precessional to axial motion, J. Org. Chem., 2014, 79, 3587-3600. 